\title{
Mycoplasma genitalium among Young, Urban Pregnant Women
}

\author{
Vanessa L. Short, ${ }^{1}$ Jørgen S. Jensen, ${ }^{2}$ Deborah B. Nelson, ${ }^{3}$ Pamela J. Murray, ${ }^{4}$ \\ Roberta B. Ness, ${ }^{5}$ and Catherine L. Haggerty ${ }^{1}$ \\ ${ }^{1}$ Department of Epidemiology, Graduate School of Public Health, University of Pittsburgh, Pittsburgh, PA 15261, USA \\ ${ }^{2}$ Mycoplasma Laboratory, Statens Serum Institut, DK-2300 Copenhagen, Denmark \\ ${ }^{3}$ Department of Public Health, Temple University, Philadelphia, PA 19122, USA \\ ${ }^{4}$ Department of Pediatrics, University of Pittsburgh School of Medicine, Pittsburgh, PA 15261, USA \\ ${ }^{5}$ The University of Texas School of Public Health, Houston, TX 77225, USA
}

Correspondence should be addressed to Vanessa L. Short, c-vshort@state.pa.us

Received 24 November 2009; Revised 24 February 2010; Accepted 24 February 2010

Academic Editor: Jane R. Schwebke

Copyright ( $) 2010$ Vanessa L. Short et al. This is an open access article distributed under the Creative Commons Attribution License, which permits unrestricted use, distribution, and reproduction in any medium, provided the original work is properly cited.

\begin{abstract}
Objective. As the consequences of Mycoplasma genitalium in pregnant women are unknown, we examined the relationship between prenatal M. genitalium infection and SAB. Methods. The presence of M. genitalium was determined by PCR in urine from 82 women who subsequently experienced a SAB and 134 women who maintained their pregnancies past 22 weeks gestation. The relationships between M. genitalium and subsequent SAB, demographic, current pregnancy, and reproductive health history characteristics were evaluated. Results. Compared to women without M. genitalium, women with M. genitalium were more likely to report nulliparity ( $41.7 \%$ versus $17.4 \%, P=.04)$, history of pelvic inflammatory disease $(27.3 \%$ versus $8.8 \%, P=.08)$, prior $C$. trachomatis infection $(63.6 \%$ versus $36.9 \%, P=.11$, ) and problems getting pregnant $(18.2 \%$ versus $4.4 \%, P=.10)$. M. genitalium was not associated with SAB (AOR 0.9, 95\% CI 0.2-3.8). Conclusions. Pregnant women who test positive for M. genitalium do not have an increased risk of SAB but report a history of reproductive morbidities.
\end{abstract}

\section{Introduction}

Spontaneous abortion $(\mathrm{SAB})$, the loss of a conceptus prior to 20 weeks, is the most common adverse outcome of pregnancy, occurring in an estimated $15 \%$ of clinically recognized pregnancies [1] and up to $50 \%$ of all pregnancies [2]. Many SABs occurring in the first trimester are due to phenotypic and/or chromosomal abnormalities, while environmental factors may have a greater impact on SAB occurring in later pregnancy [2]. Evidence suggests that sexually transmitted bacterial and viral infections, such as Treponema pallidum, bacterial vaginosis (BV), and Chlamydia trachomatis, may play a role in SAB [3-13].

Mycoplasma genitalium, a sexually transmitted bacterium, has been linked to various adverse gynecologic and reproductive events. It has been associated with cervicitis [14-17] and has been implicated as an etiological agent of pelvic inflammatory disease (PID) independent of C. trachomatis and Neisseria gonorrhoeae [17-20]. Furthermore, it has been detected in cervical and salpingeal samples from women with laparoscopically confirmed salpingitis [21] and in cervical and endometrial specimens from women with histologically confirmed endometritis [18]. A serologic relationship between $M$. genitalium and tubal factor infertility has also been identified $[22,23]$.

Because M. genitalium has been associated with these morbidities, it is plausible that $M$. genitalium can infect the upper genital tract during pregnancy, resulting in adverse pregnancy outcomes. However, data on M. genitalium in pregnancy are sparse. Whereas two studies among low-risk women have reported no relationship between M. genitalium and $\mathrm{SAB}[11,24]$, one study has reported an association between M. genitalium and preterm birth [25]. Thus, it is possible that M. genitalium may have an adverse effect on pregnancy outcomes. We conducted a nested case-control study to examine the role of $M$. genitalium in early pregnancy and subsequent SAB. The associations between M. genitalium and microbiologic, demographic, current pregnancy, 
and reproductive health history characteristics among predominantly young, single, African-American women with a history of sexually transmitted diseases (STDs) were also investigated.

\section{Materials and Methods}

2.1. Study Population. For our analyses, we used data from participants enrolled in the prospective Early Pregnancy Study (EPS), designed to examine the influence of violence on SAB risk [26]. During the period of January 1999 through August 2001, adolescent girls and women aged 14 to 40 years who presented to the emergency department (ED) of the hospital of the University of Pennsylvania with varying complaints, including pregnancy-related complications such as vaginal bleeding or abdominal pain, and nonpregnancyrelated complaints related to infection, accidents, and injury, were screened for eligibility [26]. Nurse interviewers administered a brief screening questionnaire to identify all pregnant women less than 22 weeks gestation seen in the ED regardless of the reason for the visit. Among the 1,249 pregnant adolescent and adult women who were eligible for the study, $96 \%$ agreed to participate $(n=1,199)$. The mean gestational age at enrollment was 10.3 weeks. All participants provided written informed consent, and the protocol and consent forms were approved by the institutional review board of the University of Pennsylvania.

2.2. Data Collection. At enrollment, nurse interviewers administered an in-person questionnaire. The interview contained information regarding medical and reproductive history, sociodemographic factors, current level of social support, living arrangements, and complications of the pregnancy. Hair samples were collected to measure cocaine use, and mid-stream urine samples were collected to assess recent tobacco and alcohol use. Follow-up telephone interviews were conducted at 16 and 22 weeks of gestation to determine pregnancy status, and medical record reviews were conducted to confirm pregnancy outcome. The 807 women who remained pregnant through 22 weeks of gestation were classified as controls (67\%), and the 392 women who experienced a noninduced pregnancy loss before 22 weeks of gestation were classified as cases (33\%). Of the cases, 212 experienced a SAB at enrollment and 180 experienced a SAB during the follow-up period.

For our subsequent EPS subanalyses, women who were experiencing a $\mathrm{SAB}$ at enrollment and women who did not have a urine sample available for analysis were excluded. Therefore, cases consisted of women who experienced a SAB during the follow-up period and had a frozen urine sample available for analysis $(n=95)$. We did a $2: 1$ match for controls and randomly selected women who did not experience a $\mathrm{SAB}$ and who had urine samples available for testing $(n=190)$. Demographic and patient characteristics between women with and without available samples did not differ significantly.

2.3. Detection of M. genitalium and C. trachomatis. In 2009, for this subsequent study, previously collected urine samples stored at $-70^{\circ} \mathrm{C}$ were tested for M. genitalium and $C$. trachomatis. M. genitalium PCR testing has been conducted in other studies stored frozen specimens for up to eight years $[19,27,28]$. Of the 285 samples sent for analysis, 216 samples (82 cases and 134 controls) were sufficient for analysis. M. genitalium was detected by an inhibitorcontrolled TaqMan real-time PCR detecting a conserved part of the MgPa adhesion gene (MG192) [29]. All positive results were confirmed by a second PCR with primers deduced from the 16 S rRNA gene sequence of M. genitalium [29]. C. trachomatis DNA was detected by an inhibitor-controlled TaqMan real-time PCR amplifying a sequence of the 16S rRNA gene of chlamydia species with a $C$. trachomatis specific TaqMan MGB probe [29]. Positive results were confirmed by a real-time PCR assay detecting a sequence of the cryptic plasmid. The methods for PCR detection of M. genitalium and $C$. trachomatis in urine have been previously validated [30].

2.4. Microbiologic, Demographic, Current Pregnancy, and Reproductive Health History Variables. The following is a list of microbiologic, demographic, current pregnancy, and reproductive health history variables that were compared between cases and controls: M. genitalium and C. trachomatis infection detected by PCR, age, race, level of education, marital status, recipient of government assistance, gestational age at enrollment, vaginal bleeding during pregnancy, parity, history of STDs, history of PID, history of problems getting pregnant, history of $\mathrm{SAB}$, prior ectopic pregnancies, and alcohol, cigarette, marijuana, and crack/cocaine use during current pregnancy. All variables were self-reported during the baseline interview. Cocaine use was additionally measured in hair specimens as previously described [26]. For our analysis, we defined cocaine use as cocaine detected in hair specimen collected at baseline and/or self-reported cocaine use. Gestational age was calculated on the basis of self-reported date of the last menstrual period.

2.5. Statistical Methods. The microbiologic, demographic, current pregnancy, and reproductive health history characteristics among cases and controls were compared using $t$, chi-square, and Fisher's exact tests for continuous and dichotomous variables as appropriate. Associations between M. genitalium PCR and microbiologic, demographic, current pregnancy, and reproductive health history characteristics were assessed with univariate logistic regression models. Variables significant in the univariate model $(P$-value $\leq .10)$ were adjusted for $C$. trachomatis PCR in multivariate logistic regression models. Nulliparity was further adjusted for age. Fisher's exact test was used to test for associations if there were cell sizes of less than five in the contingency table.

Logistic regressions were used to investigate the relationship between $M$. genitalium urine PCR and SAB. We had a power of $80 \%$ to detect a 3.4 increased risk of $\mathrm{SAB}$ among women infected with M. genitalium. Analyses were repeated controlling for age, history of SAB, smoking, and gestational age. Regression models were repeated without history of $S A B$, as including the history of $S A B$ variable in the model may bias the relationship toward the null if there is 
TABLE 1: Characteristics of women who experienced a SAB and women who maintained their pregnancies past 22 weeks gestation.

\begin{tabular}{|c|c|c|c|}
\hline Characteristic & $\begin{array}{l}\text { Women who experienced } \\
\text { a SAB } n=82 \\
n(\%)\end{array}$ & $\begin{array}{l}\text { Women who maintained } \\
\text { their pregnancies } n=134 \\
\qquad n(\%)\end{array}$ & $P$-value \\
\hline \multicolumn{4}{|l|}{ Infection } \\
\hline M. genitalium & $3(3.7)$ & $9(6.7)$ & .54 \\
\hline C. trachomatis & $3(3.7)$ & $12(9.0)$ & .17 \\
\hline M. genitalium and C. trachomatis & 0 & $1(0.7)$ & 1.00 \\
\hline \multicolumn{4}{|l|}{ Demographic } \\
\hline Mean age (standard deviation) & $24.8(6.2)$ & $23.9(5.7)$ & .33 \\
\hline \multicolumn{4}{|l|}{ Age } \\
\hline$<25$ years & $49(59.7)$ & $92(68.7)$ & .18 \\
\hline$\geq 25$ years & $33(40.2)$ & $42(31.3)$ & \\
\hline \multicolumn{4}{|l|}{ Race/ethnicity } \\
\hline African-American & $72(88.9)$ & $120(89.6)$ & .89 \\
\hline Other & $9(11.1)$ & $14(10.4)$ & \\
\hline \multicolumn{4}{|l|}{ Marital Status } \\
\hline Unmarried & $68(82.9)$ & $107(79.8)$ & .57 \\
\hline Married & $14(17.1)$ & $27(20.2)$ & \\
\hline \multicolumn{4}{|l|}{ Education } \\
\hline$<$ High school & $25(30.5)$ & $50(37.3)$ & .31 \\
\hline$\geq$ High school & $57(69.5)$ & $84(62.7)$ & \\
\hline Receiving government assistance & $31(37.8)$ & $58(43.3)$ & .43 \\
\hline \multicolumn{4}{|l|}{ Current pregnancy history } \\
\hline $\begin{array}{l}\text { Mean gestational age }{ }^{(\mathrm{a})} \text { (standard } \\
\text { deviation) }\end{array}$ & $8.7(3.2)$ & $9.5(3.4)$ & .08 \\
\hline Vaginal bleeding at enrollment & $47(57.3)$ & $29(21.8)$ & $<.0001$ \\
\hline Vaginal bleeding since LMP & $53(65.4)$ & $50(37.3)$ & $<.0001$ \\
\hline Alcohol use & $30(36.6)$ & $44(32.8)$ & .57 \\
\hline Cigarette smoking & $31(38.3)$ & $44(32.8)$ & .42 \\
\hline Marijuana use & $17(21.0)$ & $25(18.7)$ & .68 \\
\hline Crack/cocaine use $\mathrm{e}^{(\mathrm{b})}$ & $16(25.4)$ & $21(22.8)$ & .71 \\
\hline \multicolumn{4}{|l|}{ Reproductive health history } \\
\hline Nulliparous & $14(17.1)$ & $27(20.1)$ & .58 \\
\hline Prior SAB & $34(40.2)$ & $35(26.1)$ & .03 \\
\hline Prior ectopic pregnancy & $5(7.3)$ & $6(5.6)$ & .75 \\
\hline History of STD ${ }^{(c)}$ & $48(60.0)$ & $82(62.1)$ & .76 \\
\hline History of PID & $6(7.4)$ & $15(11.3)$ & .36 \\
\hline History of problems getting pregnant & $5(6.3)$ & $6(4.5)$ & .75 \\
\hline
\end{tabular}

Note: Missing observations: race, $n=1$; vaginal bleeding, $n=1$; cigarette smoking, $n=1$; marijuana use, $n=1$; crack/cocaine use, $n=61$; prior incompetent cervix, $n=2$; prior ectopic pregnancy, $n=41$; history of STD, $n=5$; history of PID, $n=3$; history of problems getting pregnant, $n=2$.

(a) Gestational age at enrollment in weeks.

(b) Detected in hair sample collected at enrollment and/or self-report cocaine use.

${ }^{(c)}$ C. trachomatis, N. gonorrhoeae, syphilis, genital warts, Trichomonas vaginalis, and/or BV.

an association between $M$. genitalium and SAB. We repeated our analyses categorizing SAB by gestational age ( $<11$ weeks, $\geq 11$ weeks), since gestational age of 11 weeks or greater at time of SAB can be used as a marker for when pregnancies are more likely to be chromosomally normal and other factors such as infectious agents are more likely to cause pregnancy loss [2]. We also repeated our analyses excluding women who experienced vaginal bleeding at enrollment, as these women may have already been experiencing a SAB and this may have compromised temporality.

\section{Results}

Among all study participants, 5.6\% (12/216) tested positive for $M$. genitalium and 6.9\% (15/216) tested positive for $C$. trachomatis at enrollment. Only one woman was coinfected 
TABLE 2: Characteristics of study participants according to M. genitalium PCR result.

\begin{tabular}{|c|c|c|c|}
\hline Characteristic & $\begin{array}{l}\text { M. genitalium positive } n=12 \\
n(\%)\end{array}$ & $\begin{array}{l}\text { M. genitalium negative } n=207 \\
\qquad n(\%)\end{array}$ & $P$-value \\
\hline \multicolumn{4}{|l|}{ Coinfection } \\
\hline \multicolumn{4}{|l|}{ C. trachomatis } \\
\hline Yes & $1(8.3)$ & $14(6.8)$ & .83 \\
\hline No & $11(91.7)$ & $193(93.2)$ & \\
\hline \multicolumn{4}{|l|}{ Demographic } \\
\hline \multicolumn{4}{|l|}{ Age } \\
\hline$<25$ years & $10(83.3)$ & $133(64.2)$ & .22 \\
\hline$\geq 25$ years & $2(16.7)$ & $74(35.8)$ & \\
\hline \multicolumn{4}{|l|}{ Race/ethnicity } \\
\hline African American & $11(91.7)$ & $184(89.3)$ & .80 \\
\hline White/Other & $1(8.3)$ & $22(10.7)$ & \\
\hline \multicolumn{4}{|l|}{ Marital status } \\
\hline Unmarried & $12(100)$ & $166(80.2)$ & — $^{\text {(a) }}$ \\
\hline Married & 0 & $41(19.8)$ & \\
\hline \multicolumn{4}{|l|}{ Education } \\
\hline$<$ High school & $5(41.7)$ & $72(34.8)$ & .63 \\
\hline$\geq$ High school & $7(58.3)$ & $135(65.2)$ & \\
\hline \multicolumn{4}{|l|}{ Current pregnancy history } \\
\hline $\begin{array}{l}\text { Mean gestational age }{ }^{(\mathrm{b})} \text { (standard } \\
\text { deviation) }\end{array}$ & $9.5(2.9)$ & $9.2(3.3)$ & $.78^{(\mathrm{c})}$ \\
\hline \multicolumn{4}{|l|}{ Vaginal bleeding at enrollment } \\
\hline Yes & $4(33.3)$ & $74(35.9)$ & 1.00 \\
\hline No & $8(66.7)$ & $132(64.1)$ & \\
\hline \multicolumn{4}{|l|}{ Vaginal bleeding since LMP } \\
\hline Yes & $5(41.7)$ & $101(49.0)$ & .62 \\
\hline No & $7(58.3)$ & $105(51.0)$ & \\
\hline \multicolumn{4}{|l|}{ Alcohol use } \\
\hline Yes & $5(41.7)$ & $70(33.8)$ & .87 \\
\hline No & $7(58.3)$ & $137(66.2)$ & \\
\hline \multicolumn{4}{|l|}{ Cigarette use } \\
\hline Yes & $6(50.0)$ & $71 / 206(34.5)$ & .28 \\
\hline No & $6(50.0)$ & $135 / 206(65.5)$ & \\
\hline \multicolumn{4}{|l|}{ Marijuana use } \\
\hline Yes & $4(33.3)$ & $40 / 206(19.4)$ & .27 \\
\hline No & $8(66.7)$ & $166 / 206(80.6)$ & \\
\hline \multicolumn{4}{|l|}{ Crack/cocaine use $\mathrm{d}^{(\mathrm{d})}$} \\
\hline Yes & $2(20.0)$ & $36(24.3)$ & .75 \\
\hline No & $8(80.0)$ & $112(75.1)$ & \\
\hline \multicolumn{4}{|l|}{ Reproductive health history } \\
\hline \multicolumn{4}{|l|}{ Nulliparous } \\
\hline Yes & $5(41.7)$ & $36(17.4)$ & .04 \\
\hline No & $7(58.3)$ & $171(82.6)$ & \\
\hline \multicolumn{4}{|l|}{ History of SAB } \\
\hline Yes & $1(8.3)$ & $68(32.8)$ & .07 \\
\hline No & $11(91.7)$ & $139(67.2)$ & \\
\hline \multicolumn{4}{|l|}{ Prior ectopic pregnancy } \\
\hline Yes & $0(0)$ & $11(6.4)$ & - $^{(\mathrm{a})}$ \\
\hline No & $7(100)$ & $160(93.6)$ & \\
\hline
\end{tabular}


TABle 2: Continued.

\begin{tabular}{lcc}
\hline Characteristic & $\begin{array}{c}\text { M. genitalium positive } n=12 \\
n(\%)\end{array}$ & $\begin{array}{c}\text { M. genitalium negative } n=207 \\
n(\%)\end{array}$ \\
\hline $\begin{array}{l}\text { History of STD }{ }^{(e)} \\
\text { Yes }\end{array}$ & $11(100)$ & $121(59.6)$ \\
No & $0(0)$ & $82(40.4)$ \\
History of C. trachomatis & & $76(36.9)$ \\
Yes & $7(63.6)$ & $130(63.1)$ \\
No & $4(36.4)$ & $18(8.8)$ \\
History of PID & & $187(91.2)$ \\
Yes & $3(27.3)$ & .11 \\
No & $7(72.7)$ & $9(4.4)$ \\
History of problems getting pregnant & & $197(95.6)$ \\
Yes & $2(18.2)$ & .08 \\
No & $9(81.8)$ & .10 \\
\hline
\end{tabular}

Note: Missing observations: race, $n=1$; vaginal bleeding, $n=1$; cigarette smoking, $n=1$; marijuana use, $n=1$; crack/cocaine use, $n=61$; prior incompetent cervix, $n=2$; prior ectopic pregnancy, $n=41$; history of STD, $n=5$; history of PID, $n=3$; history of problems getting pregnant, $n=2$.

(a) $P$-value not calculated.

(b) Gestational age at enrollment in weeks.

(c) $t$-test $P$-value $=.78$.

(d) Detected in hair sample collected at enrollment and/or self-reported cocaine use.

${ }^{(e)}$ C. trachomatis, N. gonorrhoeae, syphilis, genital warts, Trichomonas vaginalis, and/or BV.

TABLE 3: Odds of spontaneous abortion according to infection at enrollment.

\begin{tabular}{|c|c|c|c|c|}
\hline Infection & $\begin{array}{c}\text { Women who experienced a } \\
\text { SAB } n=82 \\
n(\%)\end{array}$ & $\begin{array}{l}\text { Women who maintained their } \\
\text { pregnancies } n=134 \\
n(\%)\end{array}$ & OR (95\% CI) & $\begin{array}{l}\mathrm{AOR}(95 \% \\
\mathrm{CI})^{(\mathrm{a})}\end{array}$ \\
\hline \multicolumn{5}{|c|}{ M. genitalium } \\
\hline Yes & $3(3.7)$ & $9(6.7)$ & $0.5(0.1-2.0)$ & $0.9(0.2-3.8)$ \\
\hline No & $79(96.3)$ & $125(93.3)$ & & \\
\hline
\end{tabular}

(a) Adjusted for age, history of SAB, smoking, and gestational age.

with both bacteria. Participants were predominantly less than 30 years old (82\%), African-American (90\%), not married (81\%), and receiving government assistance at time of enrollment (59\%). Nearly two-thirds of the women (62\%) reported a history of STDs. The mean gestational age at enrollment was 9.2 weeks.

Compared to control women, cases were significantly more likely to report vaginal bleeding both within 24 hours of the enrollment visit $(57.3 \%$ versus $21.8 \%, P<.0001$ ) and at any time during pregnancy $(65.4 \%$ versus $37.3 \%$, $P<.0001$ ) (Table 1). Cases were also significantly more likely to report a previous $\mathrm{SAB}(40.2 \%$ versus $26.1 \%, P=.03)$ than controls. These were the only characteristics that differed between cases and controls. Results remained the same after excluding women with vaginal bleeding at baseline.

Compared to women who tested negative for M. genitalium, women who tested positive for M. genitalium were more likely to report nulliparity $(41.7 \%$ versus $17.4 \%, P=.04)$, a history of PID (27.3\% versus $8.8 \%, P=.08)$, prior $C$. trachomatis infection $(63.6 \%$ versus $36.9 \%, P=.11)$, and problems getting pregnant $(18.2 \%$ versus $4.4 \%, P=.10)$ (Table 2). Although results were not statistically significant, the direction of the association remained the same after adjusting for $C$. trachomatis PCR (nulliparity AOR 3.4, 95\% CI 1.0-11.6; history of PID AOR 3.9, 95\% CI 0.9-16.1; prior C. trachomatis infection AOR 3.0, 95\% CI 0.8-10.5; problems getting pregnant AOR 4.8, 95\% CI 0.9-25.7). After adjusting for age, nulliparous women were still more likely to test positive for M. genitalium than women who did not report nulliparity, but the results were not statistically significant (AOR 2.8, 95\% CI 0.8-9.8).

M. genitalium was not independently associated with $\mathrm{SAB}$, after adjusting for age, history of $\mathrm{SAB}$, smoking during pregnancy, and gestational age (AOR 0.9, 95\% CI $0.2-$ 3.8) (Table 3). Results were similar after further adjusting for self-reported BV (AOR 0.4, 95\% CI 0.08-1.9) and after excluding women who experienced vaginal bleeding at enrollment (OR 0.4, 95\% CI 0.1-3.8). When history of SAB was removed from the multivariate model, M. genitalium was not associated with SAB. 
Nearly 28\% (22/79) of the cases experienced a SAB at less than 11 weeks gestation. Among these cases, none tested positive for $M$. genitalium. Among the cases that experienced a SAB at 11 or greater weeks of gestation, $4.3 \%(2 / 46)$ tested positive for M. genitalium. M. genitalium was not associated with SAB (OR 0.6, 95\% CI 0.1-3.0), even after adjusting for age, history of $\mathrm{SAB}$, smoking and gestational age at enrollment (AOR 1.0, 95\% CI 0.2-6.5)

\section{Disscusion}

To our knowledge, ours was the first study to prospectively evaluate the relationship between $M$. genitalium and subsequent $\mathrm{SAB}$ among a high-risk pregnant population and we found that M. genitalium in pregnancy was not associated with subsequent SAB. Our null findings are consistent with those of two other studies of $M$. genitalium and SAB. Oakeshott et al. used PCR to test urine samples from 915 pregnant women presenting at prenatal care at less than 10 weeks gestation in general practice and family planning clinics in London [11]. Only $0.66 \%$ of the samples tested positive for M. genitalium [11]. Women who experienced a subsequent $\mathrm{SAB}$ were not more likely to test positive for $M$. genitalium compared to the women who did not experience a SAB (1\% versus $0.6 \%, P=N S)$ [11]. Although the rate of $M$. genitalium was higher among $\mathrm{SAB}$ cases in this study, the power to detect a significant difference was likely limited, due to the small number of women testing positive for M. genitalium $(n=6)$ [11]. In a case-control study of 1014 recently pregnant women in Guinea-Bissau, M. genitalium detected by PCR in cervical samples collected within 24 hours of a SAB or delivery of a term neonate was not associated with SAB (OR 0.61, 95\% CI 0.07-2.5) [24]. In our study, although we had a modest sample size, $M$. genitalium was more prevalent than reported in the study by Oakeshott et al. (5.6\% versus $0.66 \%)$, and we had a power of $80 \%$ to detect approximately a threefold increased risk of SAB among $M$. genitalium positive patients. Our higher prevalence might be expected, as women in our study population were probably at high risk for STDs in general, as they were predominantly young, single, AfricanAmerican, of lower socioeconomic status (SES) and reported a history of STDs. Further, in contrast to Labbe et al., who tested for $M$. genitalium at the time of SAB and delivery, we tested for $M$. genitalium prior to pregnancy loss. Therefore, unlike the previous studies of M. genitalium and $\mathrm{SAB}$, our prospectively designed study allowed us to establish a temporal relationship between early pregnancy $M$. genitalium and subsequent SAB among a high-risk pregnant population.

In our study, the clinical, demographic, and behavioral characteristics of pregnant women who tested positive for M. genitalium and women who did not were similar. Our results are similar to those reported by Labbe et al., in which M. genitalium was not associated with any demographic, behavioral, clinical, or laboratory characteristic, including age, age at sexual debut, number of sexual partners, previous pregnancies, syphilis, HIV serology, N. gonorrhoeae and T. vaginalis $(P>.05$ for all) [24]. Conversely, Oakeshott et al. found that $M$. genitalium was more common in pregnant women who were less than 20 years of age $(P=.002)$, black $(P=.034)$, single $(P=.017)$, and had lower SES $(P=.007)$ [11]. Our null findings were likely due to the fact that our study population was a fairly homogeneous group of women, which may have biased our results toward the null.

Although M. genitalium was not associated with SAB or demographic and behavioral characteristics, it was associated with a history of other gynecologic and reproductive morbidities, including history of PID, prior chlamydial infection and problems getting pregnant. Further, our results suggest an independent relationship between $M$. genitalium and these conditions, as the results remained the same even after adjusting for the presence of $C$. trachomatis. Our results are consistent with other studies that have identified M. genitalium as a possible etiologic agent of other adverse reproductive conditions, such as nongonococcal/nonchlamydial PID [17-20] and PID-associated sequelae [19], including tubal factor infertility [22, 23]. It may be that M. genitalium was not associated with SAB in our study population of pregnant women because women with $M$. genitalium go on to develop PID and subfertility and would therefore not be enrolled in our study. Results from this study support the need for further research on the role of $M$. genitalium in reproductive and adverse pregnancy outcomes. While it is suggested that M. genitalium is associated with impaired fertility, larger, prospective studies among pregnant women and women trying to conceive are needed.

There are a few limitations to recognize as part of this study. First, the small sample sizes of some of our subgroups limited statistical power. Second, the mean gestational age at enrollment was approximately 9 weeks, so earlier SABs would have been missed. Still, the gestational age ranged from 1 to 15 , so early SAB was included. Furthermore, in subgroup analyses stratified by gestational age at enrollment, results were not different. Third, the use of self-report as a means of collecting information can introduce several biases and may not be a very accurate measure. Although gestational age was calculated on the basis of self-reported date of the last menstrual period, which may over or underestimate the true date, nearly $72 \%$ of participants had their estimated due date confirmed by pelvic ultrasound. Finally, only archived mid-stream urine (MSU) samples were available for $M$. genitalium testing in our EPS substudy, and this may have limited our analyses. While there are no data on the sensitivity of $M$. genitalium PCR in MSU versus first void urine (FVU) samples, C. trachomatis studies have found that MSU had a sensitivity of $86 \%$ to $95 \%$ compared to FVU [31]. Thus, we expect that MSU $M$. genitalium PCR yielded results in our study which would be similar to those using FVU, which Jensen et al. have previously published as the most sensitive specimen type for the detection of $M$. genitalium by PCR, as compared to cervical and urethral swab specimens [30]. In a study comparing the efficacy of FVU with cervical and urethral swab specimens for detection of $M$. genitalium using inhouse inhibitor-controlled PCR assays, more infections were detected using urine samples (88\%) than cervical (71\%) and urethral swab specimens (57\%), and the urine specimen was 
significantly more efficient than both the cervical $(P=.049)$ and the urethral swab specimen $(P=.0009)$ [30]. However, for optimal sensitivity, urine should be supplemented with a cervical specimen. In our study, the addition of a cervical or vaginal specimen for PCR may have increased the sensitivity and detected more M. genitalium positive women. However, cervical samples were not available for testing. Therefore, the reported prevalence of $M$. genitalium in our study population may be an underestimate of the true prevalence in our study population. The null relationship between M. genitalium urine PCR and SAB may further be explained by the fact that some women did not have upper genital tract infection, but instead had only urinary or lower genital tract infection, insufficient to cause SAB.

\section{Conclusions}

In conclusion, among this population of mostly young, single, African-American pregnant women, M. genitalium was not predictive of SAB. However, trends between $M$. genitalium and a history of gynecologic and reproductive morbidities, including other STDs, PID, and problems getting pregnant were evident, even after adjusting for $C$. trachomatis. Results from this study support the need for further research on the role of $M$. genitalium in reproductive and adverse pregnancy outcomes. While it is suggested that M. genitalium is associated with impaired fertility, larger, prospective studies among pregnant women and women trying to conceive are needed. Such studies should consider testing for $M$. genitalium in cervical or vaginal specimens from women recruited prior to pregnancy or from women early in gestation.

\section{Acknowledgments}

This work was funded by R01 HD36918-04 from the National Institute of Child Health and Human Development and the Department of Epidemiology Small Grants Program, Department of Epidemiology, University of Pittsburgh Graduate School of Public Health.

\section{References}

[1] A. J. Wilcox, C. R. Weinberg, J. F. O'Connor, et al., "Incidence of early loss of pregnancy," The New England Journal of Medicine, vol. 319, no. 4, pp. 189-194, 1988.

[2] K. Kline, Z. Stein, and M. Susser, Conception to Birth Epidemiology of Prenatal Development, Oxford University Press, New York, NY, USA, 1989.

[3] A. Lindstrand, S. Bergstrom, A. Bugalho, G. Zanconato, A.-M. Helgesson, and B. Hederstedt, "Prevalence of syphilis infection in Mozambican women with second trimester miscarriage and women attending antenatal care in second trimester," Genitourinary Medicine, vol. 69, no. 6, pp. 431-433, 1993.

[4] K. F. Schulz, W. Cates Jr., and P. R. O’Mara, "Pregnancy loss, infant death, and suffering: legacy of syphilis and gonorrhoea in Africa," Genitourinary Medicine, vol. 63, no. 5, pp. 320-325, 1987.

[5] G. G. Donders, B. Van Bulck, J. Caudron, L. Londers, A. Vereecken, and B. Spitz, "Relationship of bacterial vaginosis and mycoplasmas to the risk of spontaneous abortion,"
American Journal of Obstetrics and Gynecology, vol. 183, no. 2, pp. 431-437, 2000.

[6] B. Guerra, T. Ghi, S. Quarta, et al., "Pregnancy outcome after early detection of bacterial vaginosis," European Journal of Obstetrics Gynecology and Reproductive Biology, vol. 128, no. 1-2, pp. 40-45, 2006.

[7] P. E. Hay, R. F. Lamont, D. Taylor-Robinson, D. J. Morgan, C. Ison, and J. Pearson, "Abnormal bacterial colonisation of the genital tract and subsequent preterm delivery and late miscarriage," British Medical Journal, vol. 308, no. 6924, pp. 295-298, 1994.

[8] J. A. McGregor, J. I. French, R. Parker, et al., "Prevention of premature birth by screening and treatment for common genital tract infections: results of a prospective controlled evaluation," American Journal of Obstetrics and Gynecology, vol. 173, no. 1, pp. 157-167, 1995.

[9] P. Oakeshott, P. Hay, S. Hay, F. Steinke, E. Rink, and S. Kerry, "Association between bacterial vaginosis or chlamydial infection and miscarriage before 16 weeks' gestation: prospective community based cohort study," British Medical Journal, vol. 325, no. 7376, pp. 1334-1336, 2002.

[10] S. G. Ralph, A. J. Rutherford, and J. D. Wilson, "Influence of bacterial vaginosis on conception and miscarriage in the first trimester: cohort study," British Medical Journal, vol. 319, no. 7204, pp. 220-223, 1999.

[11] P. Oakeshott, P. Hay, D. Taylor-Robinson, et al., "Prevalence of Mycoplasma genitalium in early pregnancy and relationship between its presence and pregnancy outcome," BJOG, vol. 111, no. 12, pp. 1464-1467, 2004.

[12] S. S. Witkin and W. J. Ledger, "Antibodies to Chlamydia trachomatis in sera of women with recurrent spontaneous abortions," American Journal of Obstetrics and Gynecology, vol. 167, no. 1, pp. 135-139, 1992.

[13] F. Licciardi, J. A. Grifo, Z. Rosenwaks, and S. S. Witkin, "Relation between antibodies to Chlamydia trachomatis and spontaneous abortion following in vitro fertilization," Journal of Assisted Reproduction and Genetics, vol. 9, no. 3, pp. 207210, 1992.

[14] L. Falk, H. Fredlund, and J. S. Jensen, "Signs and symptoms of urethritis and cervicitis among women with or without Mycoplasma genitalium or Chlamydia trachomatis infection," Sexually Transmitted Infections, vol. 81, no. 1, pp. 73-78, 2005.

[15] L. E. Manhart, C. W. Critchlow, K. K. Holmes, et al., "Mucopurulent cervicitis and Mycoplasma genitalium," Journal of Infectious Diseases, vol. 187, no. 4, pp. 650-657, 2003.

[16] J. Pepin, A.-C. Labbe, N. Khonde, et al., "Mycoplasma genitalium: an organism commonly associated with cervicitis among west African sex workers," Sexually Transmitted Infections, vol. 81, no. 1, pp. 67-72, 2005.

[17] M. Uno, T. Deguchi, H. Komeda, et al., "Mycoplasma genitalium in the cervices of Japanese women," Sexually Transmitted Diseases, vol. 24, no. 5, pp. 284-286, 1997.

[18] C. R. Cohen, L. E. Manhart, E. A. Bukusi, et al., "Association between Mycoplasma genitalium and acute endometritis," Lancet, vol. 359, no. 9308, pp. 765-766, 2002.

[19] C. L. Haggerty, P. A. Totten, S. G. Astete, et al., "Failure of cefoxitin and doxycycline to eradicate endometrial Mycoplasma genitalium and the consequence for clinical cure of pelvic inflammatory disease," Sexually Transmitted Infections, vol. 84, no. 5, pp. 338-342, 2008.

[20] I. Simms, K. Eastick, H. Mallinson, et al., "Associations between Mycoplasma genitalium, Chlamydia trachomatis and pelvic inflammatory disease," Journal of Clinical Pathology, vol. 56, no. 8, pp. 616-618, 2003. 
[21] C. R. Cohen, N. R. Mugo, S. G. Astete, et al., "Detection of Mycoplasma genitalium in women with laparoscopically diagnosed acute salpingitis," Sexually Transmitted Infections, vol. 81, no. 6, pp. 463-466, 2005.

[22] H. F. Clausen, J. Fedder, M. Drasbek, et al., "Serological investigation of Mycoplasma genitalium in infertile women," Human Reproduction, vol. 16, no. 9, pp. 1866-1874, 2001.

[23] H. F. Svenstrup, J. Fedder, S. E. Kristoffersen, B. Trolle, S. Birkelund, and G. Christiansen, "Mycoplasma genitalium, Chlamydia trachomatis, and tubal factor infertility-a prospective study," Fertility and Sterility, vol. 90, no. 3, pp. 513-520, 2008.

[24] A.-C. Labbe, E. Frost, S. Deslandes, A. P. Mendonca, A. C. Alves, and J. Pepin, "Mycoplasma genitalium is not associated with adverse outcomes of pregnancy in Guinea-Bissau," Sexually Transmitted Infections, vol. 78, no. 4, pp. 289-291, 2002.

[25] R. Edwards, R. Ferguson, L. Reyes, M. Brown, D. Theriaque, and P. Duff, "Assessing the relationship between preterm delivery and various microorganisms recovered from the lower genital tract," Journal of Maternal-Fetal and Neonatal Medicine, vol. 19, no. 6, pp. 357-363, 2006.

[26] D. B. Nelson, J. A. Grisso, M. M. Joffe, et al., "Violence does not influence early pregnancy loss," Fertility and Sterility, vol. 80, no. 5, pp. 1205-1211, 2003.

[27] L. E. Manhart, K. K. Holmes, J. P. Hughes, L. S. Houston, and P. A. Totten, "Mycoplasma genitalium among young adults in the United States: an emerging sexually transmitted infection," American Journal of Public Health, vol. 97, no. 6, pp. 11181125, 2007.

[28] B. Andersen, I. Sokolowski, L. Ostergaard, J. K. Møller, F. Olesen, and J. S. Jensen, "Mycoplasma qenitalium: prevalence and behavioural risk factors in the general population," Sexually Transmitted Infections, vol. 83, no. 3, pp. 237-241, 2007.

[29] J. S. Jensen, M. B. Borre, and B. Dohn, "Detection of Mycoplasma genitalium by PCR amplification of the 16S rRNA gene," Journal of Clinical Microbiology, vol. 41, no. 1, pp. 261266, 2003.

[30] J. S. Jensen, E. Bjornelius, B. Dohn, and P. Lidbrink, "Comparison of first void urine and urogenital swab specimens for detection of Mycoplasma genitalium and Chlamydia trachomatis by polymerase chain reaction in patients attending a sexually transmitted disease clinic," Sexually Transmitted Diseases, vol. 31, no. 8, pp. 499-507, 2004.

[31] J. K. Møller, B. Andersen, F. Olesen, T. Lignell, and L. Ostergaard, "Impact of menstrual cycle on the diagnostic performance of LCR, TMA, and PCE for detection of Chlamydia trachomatis in home obtained and mailed vaginal flush and urine samples," Sexually Transmitted Infections, vol. 75, no. 4, pp. 228-230, 1999. 


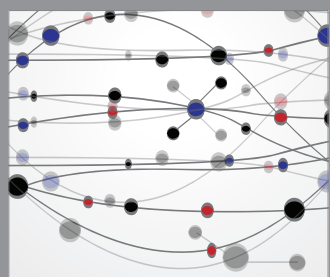

The Scientific World Journal
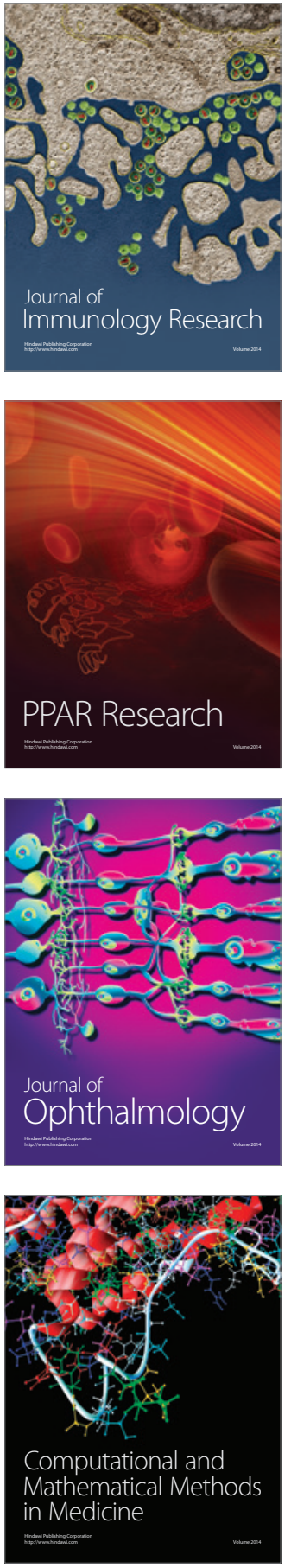

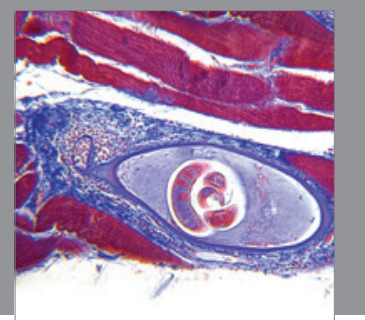

Gastroenterology

Research and Practice
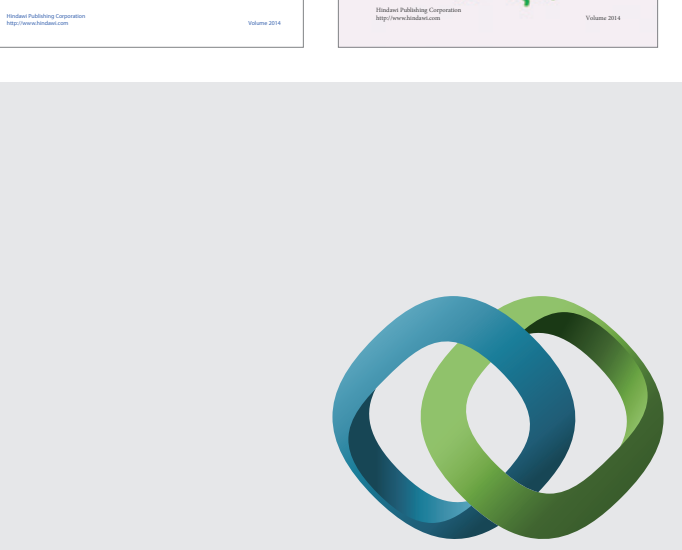

\section{Hindawi}

Submit your manuscripts at

http://www.hindawi.com
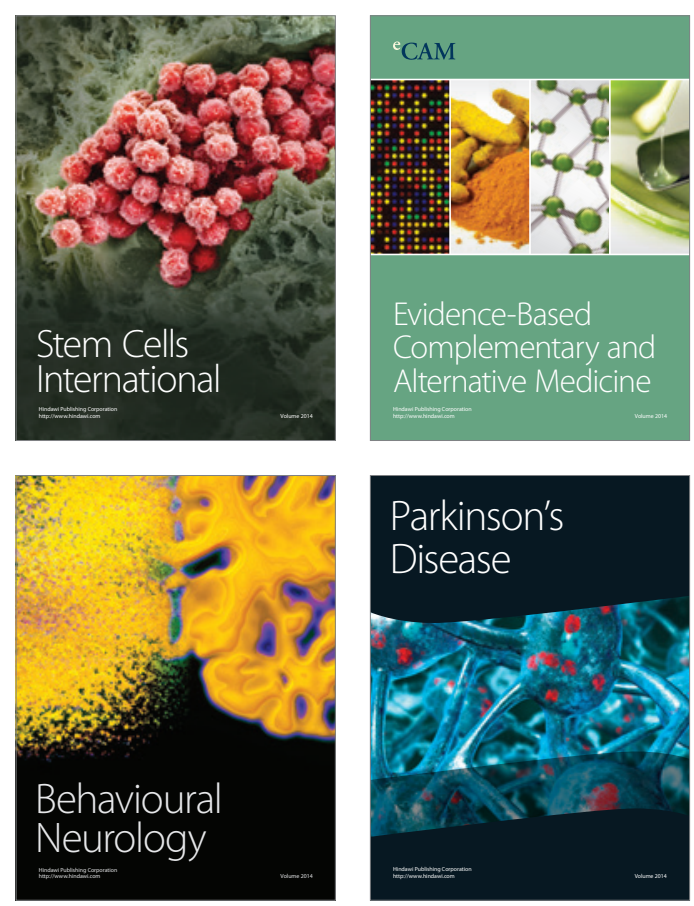

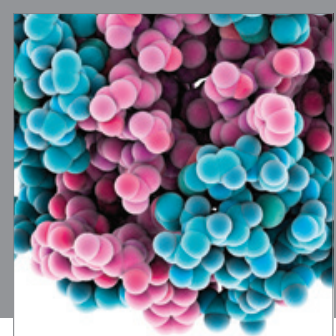

Journal of
Diabetes Research

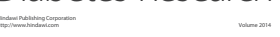

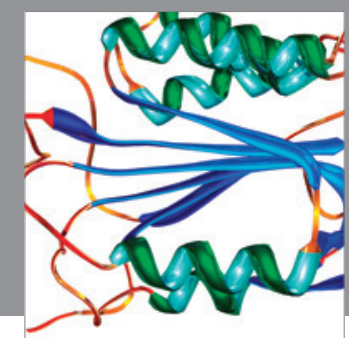

Disease Markers
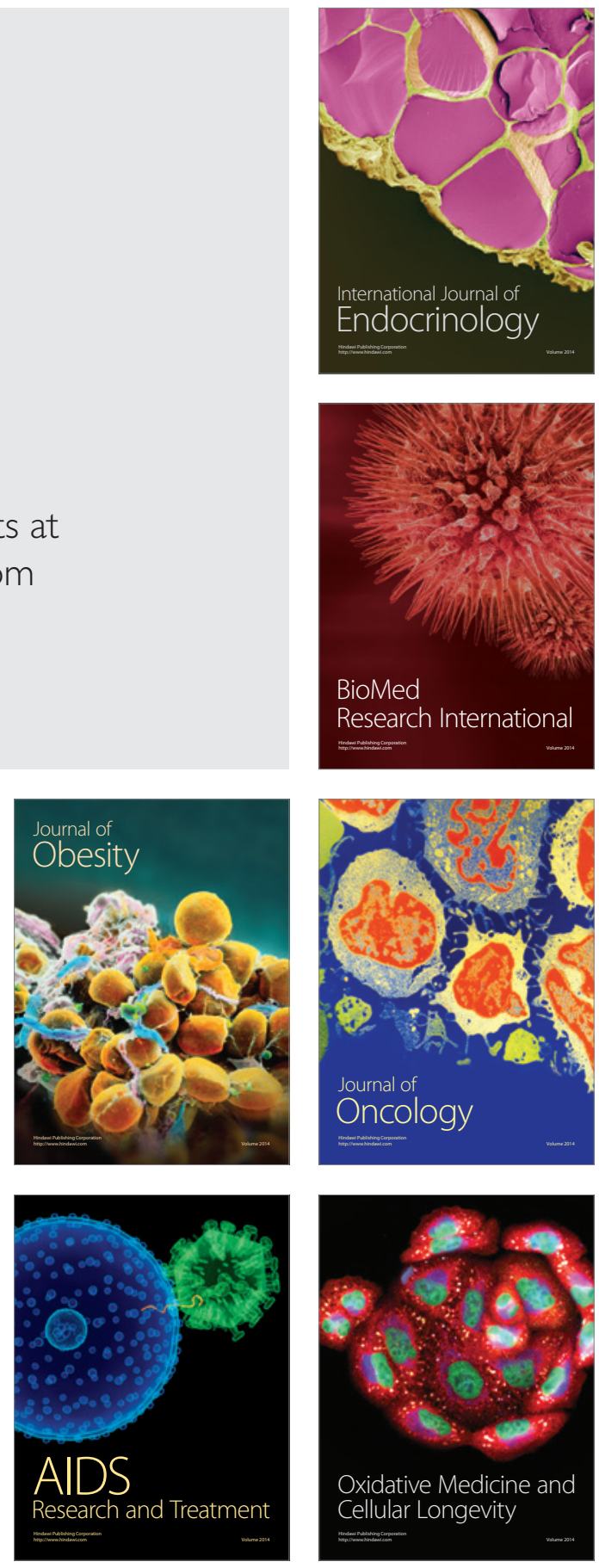